\title{
Lack of chemokine receptor CCR1 enhances Th1 responses and glomerular injury during nephrotoxic nephritis
}

\author{
Peter S. Topham, ${ }^{1,2}$ Vilmos Csizmadia, ${ }^{1}$ Dulce Soler,${ }^{1}$ Dawn Hines, ${ }^{1}$ Craig J. Gerard, ${ }^{3}$ \\ David J. Salant, ${ }^{2}$ and Wayne W. Hancock ${ }^{1,4}$ \\ ${ }^{1}$ LeukoSite Inc., Cambridge, Massachusetts 02142, USA \\ ${ }^{2}$ Renal Section, Boston University Medical Center, Boston, Massachusetts 02118, USA \\ ${ }^{3}$ Department of Medicine, Children's Hospital and Harvard Medical School, Boston, Massachusetts 02115, USA \\ ${ }^{4}$ Department of Pathology, Harvard Medical School, Boston, Massachusetts 02115, USA \\ Address correspondence to: Wayne W. Hancock, LeukoSite Inc., 215 First Street, Cambridge, Massachusetts 02142, USA. \\ Phone: (617) 621-9350 ext. 2300; Fax: (617) 621-9349; E-mail: Wayne_Hancock@leukosite.com.
}

Received for publication June 28, 1999, and accepted in revised form October 20, 1999.

During the development of nephrotoxic nephritis (NTN) in the mouse, we find that a variety of chemokines and chemokine receptors are induced: CCR1 (RANTES, MIP-1 $\alpha$ ), CCR2 (MCP-1), CCR5 (RANTES, MIP-1 $\alpha$, MIP-1 $\beta$ ), CXCR2 (MIP-2), and CXCR3 (IP-10). Their timing of expression indicated that CXCR2 and CCR1 are probably important in the neutrophil-dependent heterologous phase of the disease, whereas CCR1, CCR2, CCR5, and CXCR3 accompany the subsequent mononuclear cell infiltration characteristic of autologous disease. We therefore assessed the role of CCR1 in NTN using CCR $1^{-/-}$mice. We found that neutrophil accumulation in $C C R 1^{-/-}$mice was comparable to that in wild-type animals but that renal recruitment of $\mathrm{CD} 4^{+}$and $\mathrm{CD}^{+} \mathrm{T}$ cells and macrophages increased significantly. Moreover, $\mathrm{CCR} 1^{-/}$mice developed more severe glomerulonephritis than did controls, with greater proteinuria and blood urea nitrogen, as well as a higher frequency of crescent formation. In addition, $C C R 1^{-/-}$mice showed enhanced $\mathrm{Th} 1 \mathrm{immune}$ responses, including titers of antigen-specific IgG2a antibody, delayed-type hypersensitivity responses, and production of IFN- $\gamma$ and TNF- $\alpha$. Lastly, using recombinant proteins and transfected cells that overexpressed CCR1, we demonstrated that MIP-1 $\alpha$, but not RANTES, bound CCR1 and induced cell chemotaxis. Thus, rather than simply promoting leukocyte recruitment during NTN, CCR1 expression profoundly alters the effector phase of glomerulonephritis. Therapeutic targeting of chemokine receptors may, on occasion, exacerbate underlying disease.

J. Clin. Invest. 104:1549-1557 (1999).

\section{Introduction}

Glomerular inflammation, crescent formation, and interstitial fibrosis, the cardinal pathologic features of rapidly progressive glomerulonephritis, are mediated in large part by the infiltration of monocytes and $\mathrm{T}$ cells $(1,2)$. At least conceptually, such cellular infiltrate is thought to result from the coordinated action of multiple chemotactic factors, including leukotrienes, complement components, and chemokines (3). Recently, attention has focused on the role of chemokines in this disease. Expression of several chemokines (MIP-1 $\alpha$, MIP-1 $\beta$, MIP-2, RANTES, MCP-1, CINC, and IP-10) in rodent models of rapidly progressive glomerulonephritis is reported on (4-8), and the functional importance of individual chemokines has in some cases been determined. For example, administration of a polyclonal antiserum to MIP-2 and CINC reduced glomerular neutrophil influx and proteinuria in the heterologous phase of rat nephrotoxic nephritis (NTN) $(6,9,10)$. In an accelerated model of NTN in CD1 mice, both a RANTES antagonist (met-RANTES) and anti-MCP-1 antibodies were shown to reduce proteinuria, T-cell and macrophage infiltration, and (in the case of anti-MCP-1 antibody therapy) glomerular crescent formation and interstitial fibrosis (11). However, in a separate study of NTN in MCP-1-deficient mice, although tubulointerstitial injury was reduced, glomerular pathology was comparable to that of wildtype mice (12), highlighting some of the potential problems of data interpretation that can arise in such studies. NTN is mouse strain-dependent, different nephrotoxic sera (NTS) have variable potencies, and intervention by gene deletion or neutralization with blocking antibodies or antagonists can lead to differing outcomes. Importantly, to date, no studies have used chemokine receptor-directed strategies.

Chemokines bind to and activate $G$ protein-coupled chemokine receptors (13). The expression of different chemokine receptors by leukocyte subsets provides a further level of regulation of leukocyte trafficking, and also appears to dictate the nature of the immune response. For example, CCR5 and CXCR3 are expressed primarily by activated $\mathrm{T}$ cells and are associated with a Th1 immune response, whereas CCR 3 and CCR 4 are associated with Th2 responses (14). In this study, we analyzed the temporal expression of chemokine recep- 
tors in a mouse model of accelerated NTN, and based upon our initial data, we went on to define the role of CCR1, a receptor (at least in humans) for RANTES, MIP-1 $\alpha$, and other chemokines, using mice with a targeted disruption of this gene.

\section{Methods}

Preparation of NTS. Sheep were immunized with a lysate of glomeruli prepared from Sprague-Dawley rat kidneys, as described (15). NTS was heat-inactivated at $56^{\circ} \mathrm{C}$ for 45 minutes and then absorbed overnight with mouse red blood cells. Before use, the preparation was sterilized by passage through a $0.2-\mu \mathrm{m}$ filter.

Induction of accelerated NTN. For studies of CCR1 in glomerulonephritis, mice with a targeted disruption of the CCR1 gene $\left(C C R 1^{-/}\right)$were used. Generation of these mice has been described previously (16). They were of the same genetic background as the CCR1-sufficient $\left(C C R 1^{+/+}\right)$mice used (B6/129, intercrossed 10-20 generations). Additional control inbred C57BL/6 mice, 129 mice, and B6/129 mice were obtained from The Jackson Laboratory (Bar Harbor, Maine, USA). All mice were housed under specific pathogen-free conditions. Male $C C R 1^{+/+}$mice or $C C R 1^{-/-}$mice (each weighing approximately 30 grams) were sensitized by subcutaneous injection of $1 \mathrm{mg}$ normal sheep IgG in Freund's complete adjuvant in divided doses in each flank. Five days later, mice were injected with $0.1 \mathrm{~mL}$ of NTS daily for 3 days; control mice received normal sheep serum. At intervals from 3 to 21 days after the first dose of NTS, groups of mice (4-6 per group) were sacrificed, the kidneys were harvested, and blood was collected by cardiac puncture.

Immunopathology. Kidney halves were fixed overnight at $4{ }^{\circ} \mathrm{C}$ in $10 \%$ neutral buffered formalin and then embedded in paraffin. Four-micrometer sections were cut and then stained with periodic acid-Schiff reagent and Masson's trichrome for routine histology. All morphologic evaluations were performed in a blinded fashion using 6-8 kidneys per group for each timepoint. Glomerular pathology was assessed by evaluating at least 100 glomeruli per kidney for hypercellularity, necrosis, hyalinosis, microaneurysms, and crescent formation. Interstitial injury was assessed by determining the proportion of cortical tubules with atrophy, dilatation, or necrosis.

The remaining kidney half was snap-frozen and stored at $-70^{\circ} \mathrm{C}$ until sectioning for immunohistology. Monoclonal antibodies to mouse proteins and isotypematched control antibodies were purchased from PharMingen (San Diego, California, USA); these included monoclonal antibodies directed against all leukocytes (CD45, 30F11.1), T cells (CD4, GK1.5; and CD8, 53-6.7), monocytes and granulocytes (CD11b, M1/70), and granulocytes alone (GR-1, RB6-8C5). Cryostat sections were fixed in paraformaldehyde-lysineperiodate and stained using a 4-layer peroxidaseantiperoxidase technique (17). For assessment of glomerular cellularity, $\geq 25$ equatorially-sectioned glomeruli/kidney were examined. Results were expressed as cells per glomerular cross-section. For analysis of interstitial infiltrates, the number of positive cells per high power field (hpf; $\times 400)$ were counted $(\geq 20$ hpf/animal). For cell markers identifying dense or extensive infiltrates that precluded accurate individual cell counts, computer-aided image analysis was performed. For this purpose, $20 \mathrm{hpf} /$ animal were captured by digital video microscopy and then analyzed using IPLab software (Scanalytics Inc., Fairfax, Virginia, USA); results were expressed as percent sectional area occupied by the immunostained cells.

Renal immunoglobulin and complement deposition. Cryostat sections of renal cortical tissues (4 samples/group, taken on days 3,7 , and 14) were incubated with serial dilutions of peroxidase-conjugated rabbit anti-sheep IgG and peroxidase-conjugated rat anti-mouse IgM, IgG1, IgG2a, IgG2b, IgG2c, and C3 monoclonal antibodies (Zymed Laboratories Inc., South San Francisco, California, USA) and diaminobenzidine substrate (17). Sections were evaluated for the greatest dilution at which C3 and linear glomerular immunoglobulin deposition ( $\geq 20$ glomeruli/kidney) were still detectable.

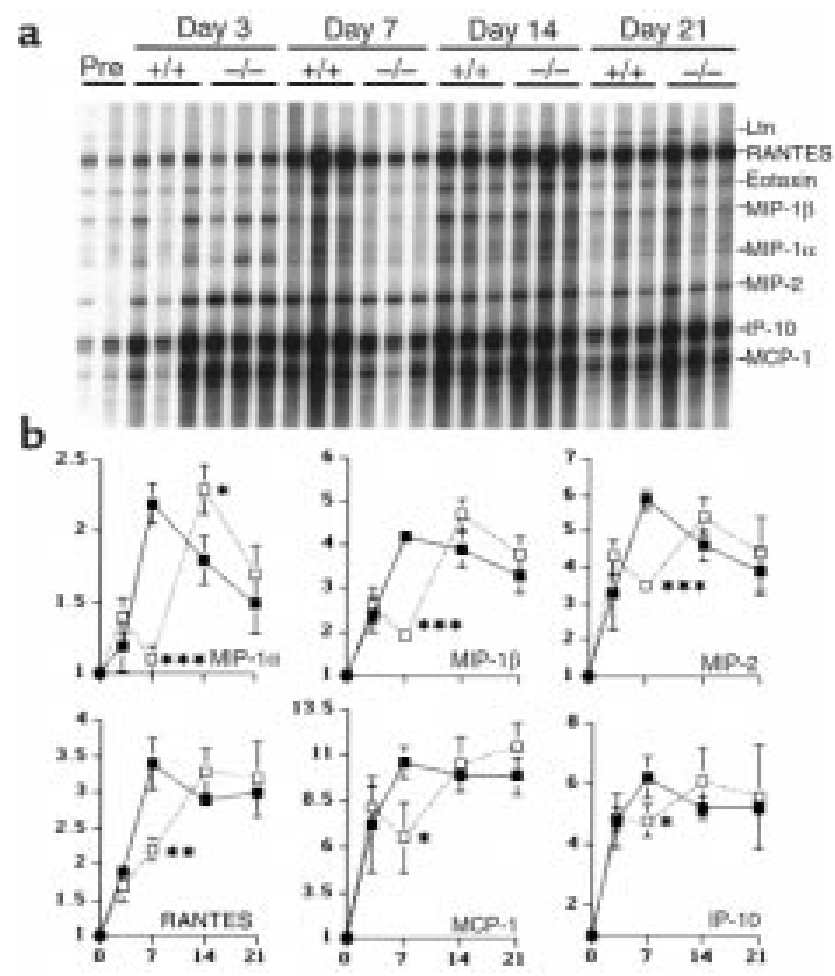

Figure 1

(a) Ribonuclease protection assay of serial renal chemokine expression in mice after NTS injection. RNA from 3 representative mice/group per timepoint were included (except for the 2 pre-NTS samples [Pre], the first of which is from a CCR $1^{+/+}$mouse and the second from a CCR1-/- mouse); the ribosomal component L32 served as a control for RNA loading. (b) The line graphs show densitometric analysis of chemokine mRNA expression after normalization to expression of the housekeeping genes $\angle 32$ and $G A P D H$. Results are expressed as fold increase over baseline expression (mean $\pm S D$ ), with statistically significant differences between $C C R 1^{+/+}$mice (filled squares) and CCR1-1- mice (open squares) evaluated by Student's $t$ test. ${ }^{*} P<0.05,{ }^{*} P<0.01,{ }^{*}{ }^{*} P<0.001$. 
Renal function. Urinary protein excretion was measured at intervals from 0 to 21 days in overnight urine collections from mice housed in individual metabolic cages. During the urine collection, mice were allowed free access to water but not food. Protein concentration was assayed using the Microprotein PR reagent kit (Sigma Chemical Co., St. Louis, Missouri, USA). Blood urea nitrogen was measured on serum samples taken at intervals from 0 to 21 days using the urea nitrogen assay kit from Sigma Chemical Co.

Ribonuclease protection assays of chemokine and chemokine receptor expression. Whole kidney RNA was isolated by guanidine-thiocyanate extraction, acid phenol/chloroform extraction, and alcohol precipitation (18); RNA integrity was confirmed by agarose gel electrophoresis and was quantitated by optical density measurement at $260 \mathrm{~nm}$. RNA from each mouse was evaluated using the RiboQuant system (PharMingen). Mouse template sets mCK5 and mCK3b were used for detection of chemokines and cytokines, respectively; template sets mCR5 and mCR6 were used to detect CC and CXC chemokine receptors, respectively. In addition, a riboprobe for mouse CXCR3 was prepared in-house. In vitro transcription was undertaken in transcription buffer supplemented with $\left[\alpha^{32} \mathrm{P}\right] \mathrm{UTP}$ (3,000 Ci/mmol; Amersham Life Sciences Inc., Arlington Heights, Illinois, USA) and T7 RNA polymerase. After DNaseI treatment, the riboprobe was isolated by phenol/chloroform extraction and precipitation with ammonium acetate and ethanol; labeling efficiency was determined by measuring Cherenkov activity in a scintillation counter. Each riboprobe set was diluted to the optimal activity defined by the manufacturer, and then added to $20 \mu \mathrm{g}$ of kidney RNA. This was heated to $90^{\circ} \mathrm{C}$, allowed to cool to $56^{\circ} \mathrm{C}$, and annealed overnight. After RNase and proteinase $\mathrm{K}$ treatment, protected RNA hybrids were purified by phenol/chloroform extraction and precipitation with ammonium acetate and ethanol, and then separated by electrophoresis on $5 \%$ polyacrylamide/8 $\mathrm{M}$ urea gels. Gels were dried and subjected to autoradiography using Kodak Biomax MS2 film (Eastman Kodak Co., Rochester, New York, USA). Autoradiographs were scanned and imported into Adobe Photoshop (Adobe Systems Inc., San Jose, California, USA). RNA bands were quantitated by densitometric analysis with NIH Image (National Institutes of Health, Bethesda, Maryland, USA), and results were normalized for $L 32$ and GAPDH gene expression.

Skin delayed-type hypersensitivity responses to sheep IgG. Mice were challenged 48 hours before the end of each experiment by intradermal injection of sheep IgG (100 $\mu \mathrm{g}$ in $20 \mu \mathrm{L}$ of PBS) into the footpad using a 30 -gauge needle. The other foot was injected with an identical volume of PBS. Footpad swelling was measured 48 hours later using a micrometer. Results are expressed as antigen-specific swelling in micrometers, calculated as the difference in thickness between vehicle- and antigen-injected footpads.

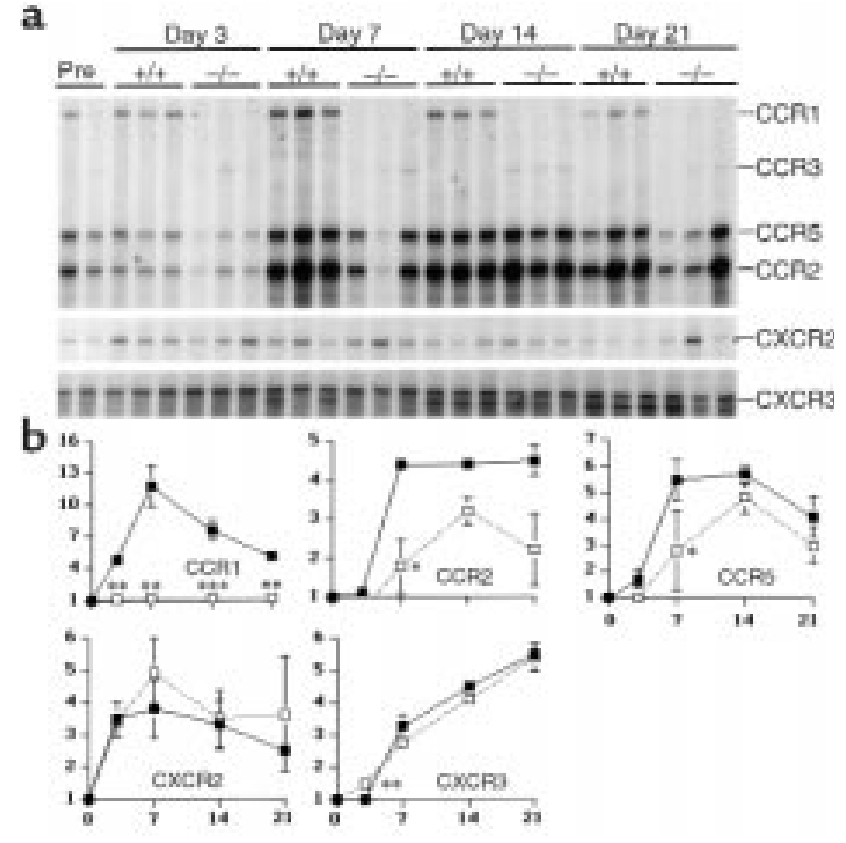

Figure 2

(a) Ribonuclease protection assay of serial renal chemokine receptor expression in mice after NTS injection. RNA from 3 representative mice/group per timepoint were included (except for the 2 pre-NTS samples, the first of which is from a $C C R 1^{+/+}$mouse and the second from a CCR1-/- mouse); the ribosomal component L32 served as a control for RNA loading. (b) The line graphs show densitometric analysis of chemokine receptor mRNA expression after normalization to expression of the housekeeping genes $\angle 32$ and GAPDH. Results are expressed as fold increase over baseline expression (mean $\pm S D$ ), with statistically significant differences between $C C R 1^{+/+}$mice (black squares) and $C C R 1^{-/-}$mice (open squares) evaluated by Student's $t$ test. ${ }^{*} P<0.05,{ }^{*} P<0.01,{ }^{*}{ }^{*} P<0.001$.

Antigen-specific cytokine production by splenic $T$ cells. Spleens from sensitized $C C R 1^{+/+}$or $C C R 1^{-/-}$mice were removed aseptically, and then placed in RPMI1640/10\% FCS medium and disrupted to form singlecell suspensions. Erythrocytes were depleted using Red Cell Lysis buffer (Sigma Chemical Co.). After washing in RPMI/FCS, each suspension was enriched for $\mathrm{T}$ cells using nylon wool columns (Polysciences Inc., Warrington, Pennsylvania, USA). Enriched T cells $\left(4 \times 10^{6}\right.$ cells $\left./ \mathrm{mL}\right)$ were incubated for 72 hours in RPMI-1640/10\% FCS in 96-well tissue culture plates with or without $20 \mu \mathrm{g} / \mathrm{mL}$ sheep IgG, and cell supernatants were assayed for IL- 4 , IFN- $\gamma$, and TNF- $\alpha$ by ELISA (Opt-EIA; PharMingen).

TNF- $\alpha$ production by splenic mononuclear cells. Single spleen cell suspensions were prepared from $C C R 1^{+/+}$or CCR1 $1^{-/-}$mice. Suspensions were then depleted of red cells and incubated in RPMI-1640/10\% FCS medium containing LPS $(1 \mu \mathrm{g} / \mathrm{mL})$ for 3 hours at $37^{\circ} \mathrm{C}$ in an atmosphere of $5 \% \mathrm{CO}_{2}$. Supernatant TNF- $\alpha$ levels were assayed by ELISA (PharMingen).

Mouse anti-sheep IgG antibody isotypes. Titers of mouse anti-sheep IgG antibody were measured by ELISA using sera collected at multiple timepoints. Microtiter plates 

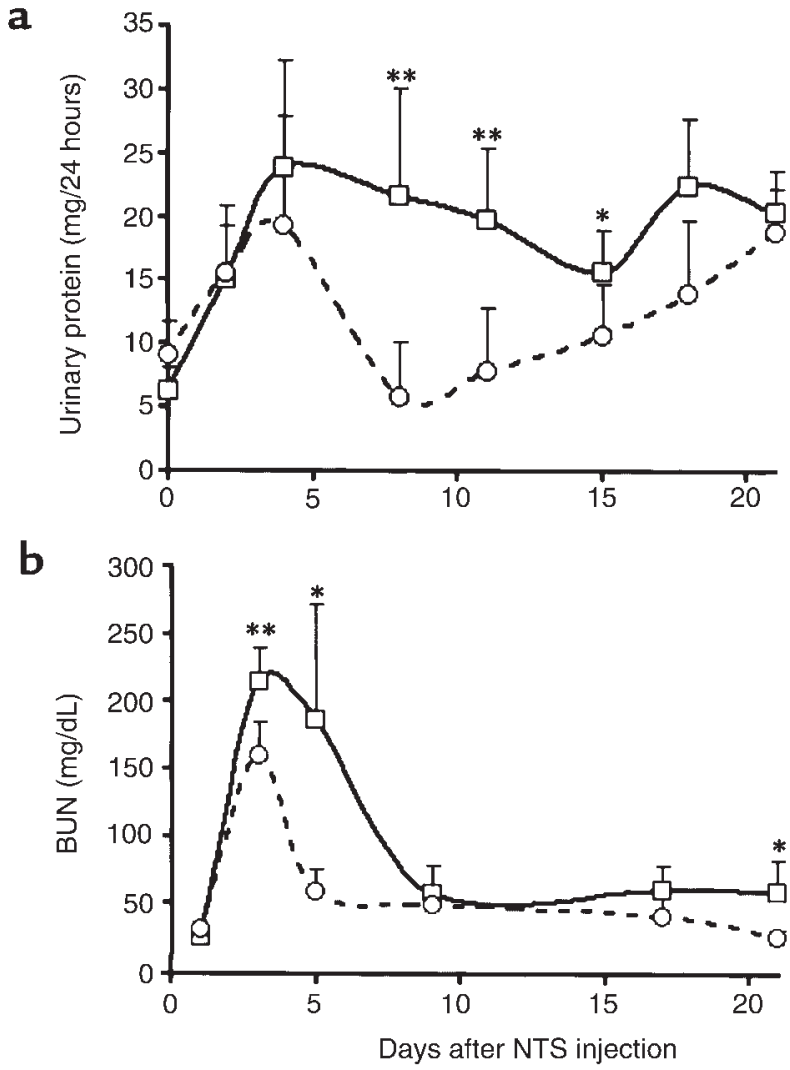

Figure 3

$C C R 1^{-/-}$mice show greater renal injury than do $C C R 1^{+/+}$mice. Renal dysfunction in mice was assessed by (a) urinary protein excretion and (b) blood urea nitrogen (BUN) concentration in $C C R 1^{+/+}$mice (open circles) and CCR $1^{-/-}$mice (open squares); mean \pm SD values for 12 animals/timepoint per group. Results of Student's $t$ test for differences between the 2 groups at a corresponding timepoint: ${ }^{*} P<0.05,{ }^{*} P<0.01$.

were coated with $100 \mu \mathrm{L}$ of $10 \mu \mathrm{g} / \mathrm{mL}$ sheep IgG in carbonate buffer ( $\mathrm{pH} 9.6)$ overnight at $4^{\circ} \mathrm{C}$. After blocking with $1 \%$ BSA in PBS, the plates were incubated with serial dilutions of mouse serum (starting at 1:100) for 2 hours at room temperature. Bound mouse IgG was detected using peroxidase-conjugated rabbit anti-mouse IgG (Jackson ImmunoResearch Laboratories Inc., Philadelphia, Pennsylvania, USA) at 1:2,000, orthopenyldiamine substrate, and absorbance readings (at $450 \mathrm{~nm}$ ) on a spectrophotometer. Lack of cross-reactivity of the secondary antibody with sheep IgG was demonstrated by omitting the primary antibody. Immunoglobulin isotypes were measured using the ELISA technique already described. Mouse serum was diluted 1:100 for IgG1 and 1:20 for IgG2a; the bound mouse immunoglobulin isotypes were detected using peroxidase-conjugated rat anti-mouse IgG1 and IgG2a antibodies (Zymed Laboratories Inc.) at a dilution of 1:2,000.

Generation of murine CCR1 L1/2 stable cell transfectants. The coding region of mCCR 1 was amplified by PCR and directionally cloned into the BamHI and NotI sites of pcDNA3 (Invitrogen Corp., San Diego, California, USA). Stable transfectant cell lines were generated in the murine pre-B lymphoma cell line $\mathrm{L} 1 / 2$ and cultured as described (19). Cells were treated with butyric acid before performing experiments. Clones selected had approximately 100,000 sites/cell after overnight stimulation with butyric acid $(10 \mathrm{mM})$, as determined by Scatchard analysis of binding data.

Chemokine synthesis. Murine RANTES and human and murine MIP- $1 \alpha$ were synthesized by f-moc chemistry (using a 433A automated peptide synthesizer; PerkinElmer Biosystems, Norwalk, Connecticut, USA). Purification and folding was carried out as described (20). The molecular integrity of each folded purified protein was determined by electron-spray mass spectrometry; all proteins had the expected specific activities. Murine recombinant MCP-3 was purchased from PeproTech Inc. (Rocky Hill, New Jersey, USA).

Chemotaxis assays. Biocoat Transwell culture inserts (3 $\mu \mathrm{m}$; Corning-Costar Corp., Acton, Massachusetts, USA) were used for 24-well chemotaxis assays. Briefly, $10^{6}$ cells in $100 \mu \mathrm{L}$ of chemotaxis medium (50\% M199, $50 \%$ RPMI-1640, and 0.5\% BSA) were dispensed into the Transwell in the chemotaxis chamber. Then $600 \mu \mathrm{L}$ of chemotaxis medium with or without chemokine was placed in the bottom well and cultures were incubated at $37^{\circ} \mathrm{C}$ for 5 hours or overnight. Cells that migrated to the bottom well were counted by flow cytometry. Relative cell counts were obtained by acquiring events for a set time of 30 seconds.

Competitive ligand binding assays. Human MIP- $1 \alpha$ labeled with ${ }^{125} \mathrm{I}$ was used as the radioligand, and mMIP- $1 \alpha$, hMIP- $1 \alpha$, mRANTES, and MMCP-3 were the cold competitive ligands. Briefly, $10^{5}$ cells were incubated for 45 minutes at ambient temperature with $0.05 \mathrm{nM}$ radiolabeled ligand in the absence or presence of increasing concentrations of unlabeled chemokine. The binding reaction was carried out in binding buffer (50 $\mathrm{mM}$ HEPES at $\mathrm{pH} 7.5,1 \mathrm{mM} \mathrm{CaCl}_{2}, 5 \mathrm{mM} \mathrm{MgCl}_{2}, 0.05 \%$ $\mathrm{Na}_{3} \mathrm{~N}$, and $0.5 \% \mathrm{BSA}$ ) in a total volume of $100 \mu \mathrm{L}$. Cells were washed 3 times with high-salt buffer (binding buffer containing $0.5 \mathrm{M} \mathrm{NaCl}$ ), and cell-associated radioactivity was assessed in a scintillation radioactivity counter. Nonspecific binding was determined in the presence of $100 \mathrm{nM}$ unlabeled chemokine. Specific binding was obtained by subtracting nonspecific binding from total binding using KaleidaGraph software (Synergy Software, Reading, Pennsylvania, USA).

\section{Results}

Chemokine and chemokine receptor $m R N A$ expression during $N T N$. In preliminary studies, several chemokine receptor mRNA types were detected within nephritic kidneys, leading to consideration of the importance of each receptor using corresponding knockout mice. This first paper deals with the effects of absence of CCR 1 on the pathogenesis of NTN; results are presented in each case with normal kidneys compared with corresponding nephritic kidneys generated in $\mathrm{CCR} 1^{+/+}$and $\mathrm{CCR} 1^{-/-}$ mice. In normal kidneys, low-level expression of RANTES, MIP-1 $\beta$, MIP-2, eotaxin, and IP-10 mRNA was 

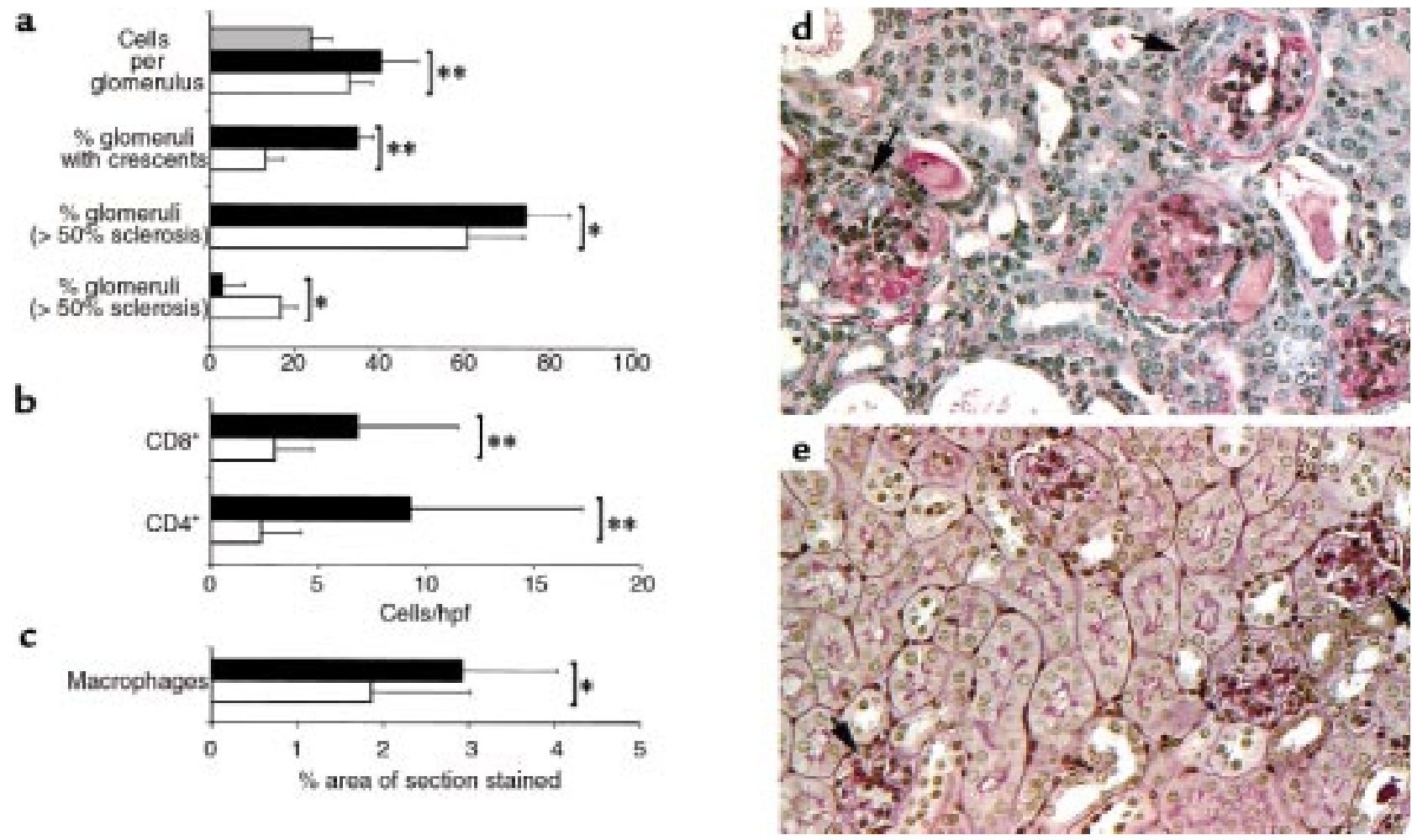

\section{Figure 4}

CCR1-/- mice show greater histologic injury than do CCR $1^{+/+}$mice, as reflected by quantitative assessment of (a) numbers of glomerular cells, crescent formation, glomerulosclerosis, and (b) T-cell and (c) macrophage accumulation in nephritic CCR $1^{+/+}$mice (open bars) vs. nephritic CCR $1^{-/-}$mice (solid bars) and normal $C C R 1^{+/+}$mice (gray bars). Assessment (as detailed in Methods) was performed 21 days after NTS injection, using 8 mice per group; results of analysis by Student's $t$ test are presented as mean $\pm \mathrm{SD}$. ${ }^{*} P<0.05$; ${ }^{*} P<0.01$ for nephritic CCR $1^{-/-}$mice vs. nephritic CCR $1^{+/+}$mice. (d and e) Representative histopathology of kidneys (8/group) harvested at day 21 after NTS from $C C R 1^{-/-}$and $C C R 1^{+/+}$mice. (d) In CCR1 $1^{-/-}$mice, most glomeruli showed extensive glomerulosclerosis and crescent formation (arrows), whereas $C C R^{+/+}$mice (e) typically showed considerably less glomerulosclerosis (arrows) and infrequent crescent formation. Paraffin sections, periodic acid-Schiff reagent counterstain, $\times 400$.

observed (Figure 1). Development of glomerular injury in $C C R 1^{+/+}$mice was associated with progressively increasing renal expression of multiple chemokine mRNAs, which typically peaked by 7 days after NTS treatment (Figure 1); at day 7, significantly increased chemokine mRNAs $(P<0.05)$ included MCP-1 (11-fold), eotaxin (8-fold), IP-10 (6-fold), MIP-2 (6-fold), MIP-1 $\beta$ (4-fold), RANTES (3-fold), and MIP-1 $\alpha$ (2-fold). TCA-3 mRNA (not shown) was unchanged during the experimental period. Chemokine mRNA data from nephritic CCR1 $1^{-1-}$ mice were generally comparable to that of $\mathrm{CCR}^{+/+}$mice, except for typically reduced mRNA expression at day 7 after NTS injection (Figure 1).

Analysis of chemokine receptor mRNA expression (Figure 2) showed serial increases of mRNA in $C C R 1^{+/+}$ mice, in most cases peaking at day 7 after NTS injection, including CCR1 (12-fold increase over baseline), CCR5 (6-fold), CCR2 (4-fold), CXCR2 (4-fold), and CXCR3 (> 3-fold). Apart from CCR1 itself, CCR1 $1^{-/-}$ mice showed decreased expression of CCR 2 and CCR5 at day 7 after NTS injection compared with $C C R 1^{+/+}$ mice (Figure 2), but comparable levels thereafter. However, with significance for future studies, levels of CXCR3 mRNA continued to rise in both groups throughout the course of study (Figure 2), whereas CCR3, CCR4, CXCR4, and CXCR5 mRNA expression remained at baseline levels.

CCR1-/- mice develop greater renal impairment than do $C C R 1^{+/+}$mice. Given that CCR1 mRNA was the most abundantly induced chemokine receptor and was expressed at all timepoints examined in nephritic but not in baseline kidneys, we compared functional parameters in $C C R 1^{-/-}$and $C C R 1^{+/+}$mice. Surprisingly, the absence of CCR1 resulted in more severe disease. In CCR1 $1^{-1-}$ mice, both urine protein excretion (21.6 mg per 24 hours at day 7 ) and blood urea nitrogen $(214 \mathrm{mg} / \mathrm{dL}$ at day 3$)$ were greater than in $C C R 1^{+/+}$ mice (respectively $5.8 \mathrm{mg}$ per 24 hours at day $7, P<$ $0.01 ; 168 \mathrm{mg} / \mathrm{dL}$ at day $3, P<0.01$ ) (Figure 3), and their elevations were more persistent (Figure 3 ). Comparable differences between CCR $1^{-/}$mice and pure C57/BL6 or 129 mice after NTS injection were also noted (data not shown).

CCR1 $1^{-1-}$ mice develop more severe crescentic glomerulonephritis than do CCR $1^{+/+}$mice. Consistent with the renal function data, the severity of histologic injury was significantly greater in $C C R 1^{-/-}$than in $C C R^{+/+}$ mice. More $C C R 1^{-/-}$mice developed crescents than did 
a
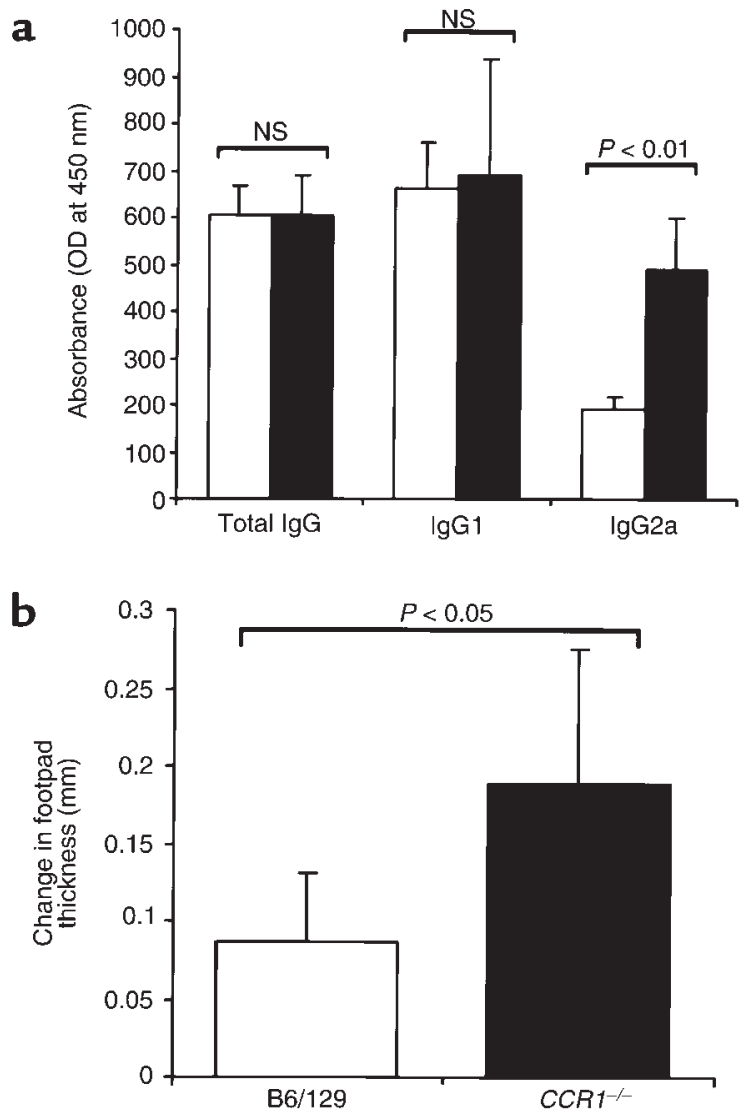

c

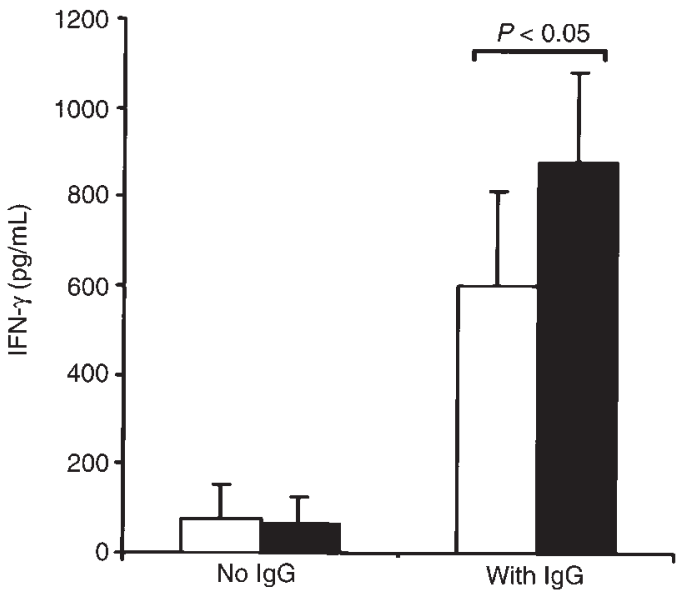

Figure 5

CCR1-/- mice display Th1 antigen-specific immune responses. (a) Circulating titers of mouse anti-sheep $\lg \mathrm{G}, \operatorname{lgG} 1$, and $\lg \mathrm{G} 2 \mathrm{a}$ isotypes at 10 days after NTS injection were measured by ELISA in nephritic CCR1 $1^{-/}$and $\mathrm{CCR} 1^{+/+}$mice. Serum dilutions were 1:200 for total $\lg \mathrm{G}$, 1:100 for $\lg \mathrm{G} 1$, and 1:20 for $\lg \mathrm{g} 2 \mathrm{a}$; results are presented as mean \pm SD of 6 animals/group as analyzed by Student's $t$ test. NS, not significant. (b) Antigen-specific DTH responses were assessed in nephritic $C C R 1^{+/+}$and $C C R 1^{-/-}$mice ( $n=10 /$ group) by injecting the hind-limb footpad with either $100 \mu \mathrm{g}$ sheep IgG or PBS and measuring footpad swelling after 48 hours; results are presented as mean \pm SD of the difference in footpad thickness between the 2 treatments, analyzed by Student's $t$ test. (c) Antigen-specific production of IFN$\gamma$ by T cell-enriched splenocytes from nephritic CCR $1^{+/+}$and $\mathrm{CCR} 1^{-/-}$ mice ( $n=10 /$ group) after 72 hours of culture in the presence or absence of $20 \mu \mathrm{g} / \mathrm{mL}$ sheep IgG. IFN- $\gamma$ was measured by ELISA; results are presented as mean \pm SD.
CCR $1^{+/+}$mice $\left(35 \%\right.$ of $C C R 1^{-/-}$mice vs. $17 \%$ of mice at day $21 ; P<0.01)$. In addition, $C C R 1^{-/-}$mice showed more glomerulosclerosis and glomerular hypercellularity, though several other parameters of glomerular injury (e.g., sclerosis, necrosis, and microaneurysm formation) and interstitial injury (not shown) were not significantly different between the 2 groups (Figure 4). However, T-cell and macrophage infiltration of both glomerular and interstitial compartments was greater in $C C R 1^{-/}$mice throughout the course of renal disease (Figure 4).

CCR1 $1^{-1-}$ mice show a normal humoral immune response to sheep IgG. Humoral responses were examined as a potential explanation for the enhanced disease in CCR $1^{-/-}$mice compared with $C R 1^{+/+}$mice. Glomerular deposition of sheep IgG, mouse IgG, and complement was comparable between the 2 groups (data not shown). Furthermore, serum titers of total mouse antisheep IgG were comparable in the 2 groups (Figure 5a).

CCR1 $1^{-1-}$ mice show a Th1 antigen-specific immune response. Antigen-specific IgG isotype production in $\mathrm{CCR}^{-/-}$mice was consistent with a Th1-deviated response. Although IgG1 titers were comparable in both groups (Figure 5a), the titers of IgG2a were significantly higher in the $C C R 1^{-/}$group $(P<0.01)$ (Figure 5a). Cutaneous delayed-type hypersensitivity $(\mathrm{DTH})$ responses upon intradermal challenge with sheep IgG were also significantly greater in CCR1-/mice than in $C C R 1^{+/+}$mice $(P<0.05)$ (Figure $\left.5 \mathrm{~b}\right)$. This led us to examine cytokine production from $\mathrm{T}$ cell-enriched spleen cell cultures. IFN- $\gamma$ production was significantly higher in CCR1 $1^{-/-}$mice than in control CCR $1^{+/+}$mice $(878 \mathrm{pg} / \mathrm{mL}$ vs. $597 \mathrm{pg} / \mathrm{mL}$, respectively; $P<0.05$ ) (Figure $5 \mathrm{c}$ ).

CCR $1^{-/-}$mice have enhanced mononuclear cell TNF- $\alpha$ production. Expression of TNF family genes was analyzed by ribonuclease protection assay. Figure 7 shows a representative autoradiograph demonstrating that TNF$\alpha$, TNF- $\beta$, and lymphotoxin- $\beta$ are expressed at higher levels in $C C R 1^{-/-}$mice than in $C C R 1^{+/+}$mice. In addition, isolated splenic mononuclear cells from CCR1 $1^{-/-}$ mice produced more TNF- $\alpha$ in response to LPS than did $C C R 1^{+/+}$mononuclear cells (Figure 6).

$M I P-1 \alpha$ is the predominant CCR1 ligand in mice. Because CCR 1 and at least 3 of its reported ligands (MIP- $1 \alpha$, MCP-3, and RANTES) are expressed in nephritic kidneys, we questioned why loss of CCR1 did not have an inhibitory effect on cell recruitment. Accordingly, the ligand specificity of mouse CCR1 was analyzed in vitro using mouse CCR1 transfectants in chemotaxis and ligand binding assays. These experiments demonstrated that mouse CCR1 transfectants were chemoattracted by mouse MIP- $1 \alpha$ but not by mouse RANTES or MCP-3 (Figure 7a), whereas in control wells, mouse RANTES induced a robust chemotactic response by mouse CCR5 transfectants and mouse MCP-3 chemoattracted mouse CCR3 transfectants (data not shown). Competitive binding assays using mouse CCR1 transfectants confirmed these results, demonstrating that mouse MIP-1 $\alpha$ 
alone was able to competitively inhibit binding of radiolabeled human MIP- $\alpha$, whereas mouse MCP-3 and mouse RANTES had no effect (Figure 7b).

\section{Discussion}

To our knowledge, this is the first comprehensive analysis of the expression patterns and functional roles of chemokine receptors in any kidney disease model. Our rationale was to identify those chemokine receptors whose temporal expression would suggest involvement in the recruitment of specific leukocyte subsets to nephritic kidneys in a way that they could then be targeted by interventional studies using monoclonal antibodies or genetically deficient mice.

The heterologous phase of mouse NTN involves neutrophil infiltration of glomeruli that begins within hours of NTS administration and lasts for several days $(21,22)$. Although this phase of disease was not our prime interest given the key role of complement at this stage, the results at day 3 do provide some information about chemokine receptors that may be involved in neutrophil recruitment. Both CCR1 and CXCR2 were upregulated by day 3 ; it is known that mouse neutrophils express both of these receptors, so it is possible that they each contribute to this neutrophil-dependent phase of glomerular disease $(23,24)$. However, no difference in the number of glomerular neutrophils was observed between CCR1 $1^{-/-}$and $C C R 1^{+/+}$mice at day 3 (data not shown), and the degree of early proteinuria was comparable in both groups. Because proteinuria during this early phase is at least in part due to neutrophil-mediated glomerular injury $(21,25)$, these observations suggest that neutrophil recruitment is not dependent on CCR1 in this model. CXCR2 is an alternate chemokine receptor expressed by neutrophils, and it was previously shown in a rat model of NTN (9) that neutralization of MIP-2 (a CXCR2 ligand) using a polyclonal antiserum decreased neutrophil recruitment and proteinuria during the heterologous phase of disease.

Our major interest in this model was the cell-mediated autologous phase of disease characterized by the development of glomerular crescents and tubulointerstitial disease in association with macrophage and T-cell recruitment to the glomeruli and interstitium. During this phase, the expression of CCR2, CCR5, and CXCR3 was increased. In addition, levels of CCR1 mRNA expression increased further over that first seen during the heterologous phase, and persisted at elevated levels throughout the course of the disease. These various chemokine receptors are expressed by various leukocytes, but especially by macrophages (CCR1, CCR2, and CCR5) and T cells (CCR1, CCR2, CCR5, and CXCR3). Again, the temporal association between expression of these receptors and the influx of effector cells suggests that these receptors may play important roles in disease progression and are therefore suitable targets for interventional studies. Studies demonstrating the effectiveness of ligand neutralization support potential roles for some of these receptors (e.g., MCP-1/CCR2; RANTES/CCR1, CCR5) in NTN $(11,12)$.
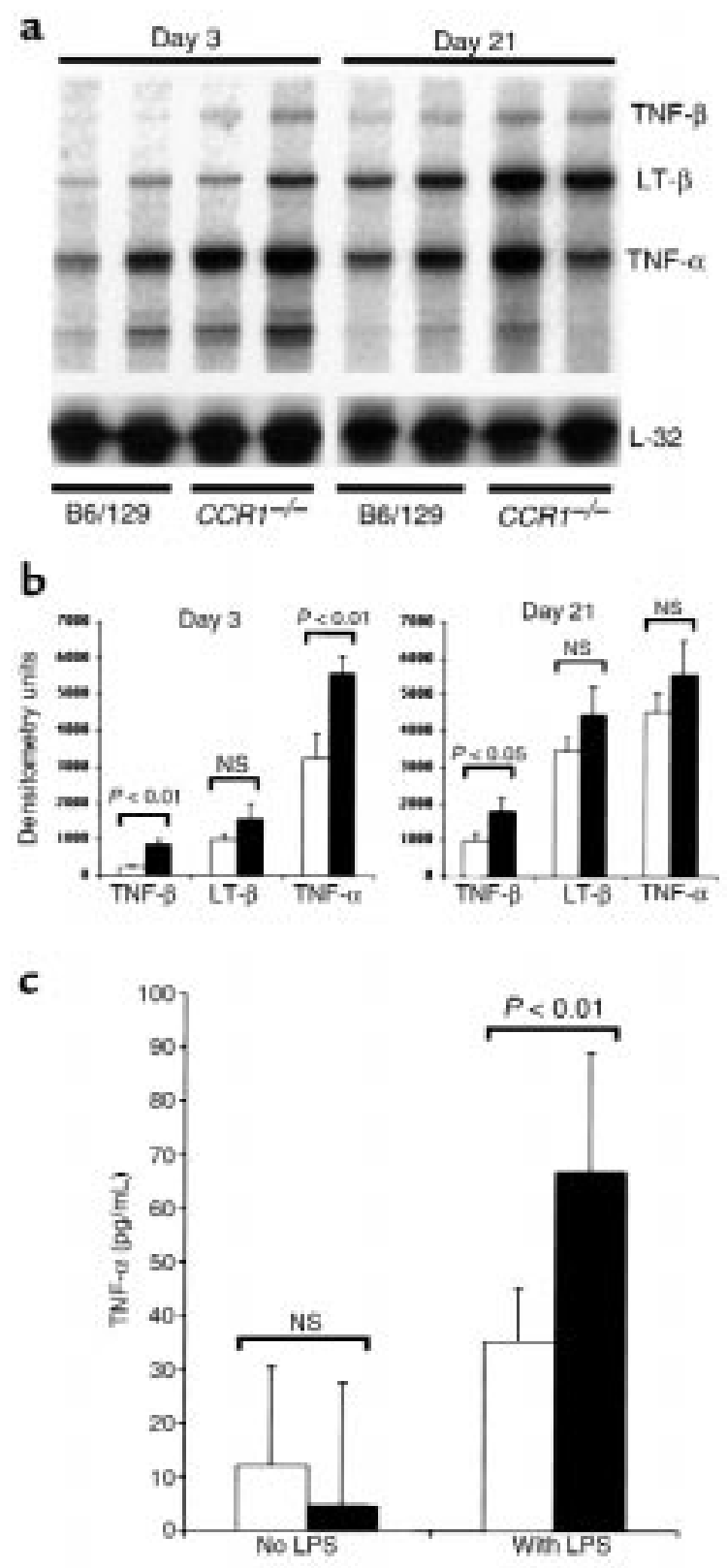

Figure 6

CCR $1^{-/-}$mice show enhanced mononuclear cell production of TNF$\alpha$. (a) Ribonuclease protection assay of renal expression of TNF- $\alpha$, TNF- $\beta$, and lymphotoxin- $\beta$ (LT- $\beta$ ) in nephritic CCR $1^{-/-}$vs. nephritic $C C R 1^{+/+}$mice at 3 days and 21 days after NTS injection. RNA loading was normalized relative to $L 32$ gene expression. (b) Densitometric analysis of TNF gene family members after data were normalized to L32 and GAPDH expression; results are presented as mean \pm SD values for 3 mice/group per timepoint (analyzed by Student's $t$ test). (c) TNF- $\alpha$ production from splenic mononuclear cells in response to LPS. Cells were isolated from $\mathrm{CCR} 1^{+/+}$and CCR $1^{-/}$mice and cultured for 3 hours; supernatants were assayed by ELISA. Results are presented as mean $\pm \mathrm{SD}$, with 4 mice/group.

CCR1 was chosen as our initial target because it is highly expressed throughout disease and might thereby promote recruitment of macrophages, $\mathrm{T}$ cells, and neutrophils; and at least 3 of its ligands (MIP-1 $1 \alpha$, RANTES, and MCP-3) are expressed during the course 

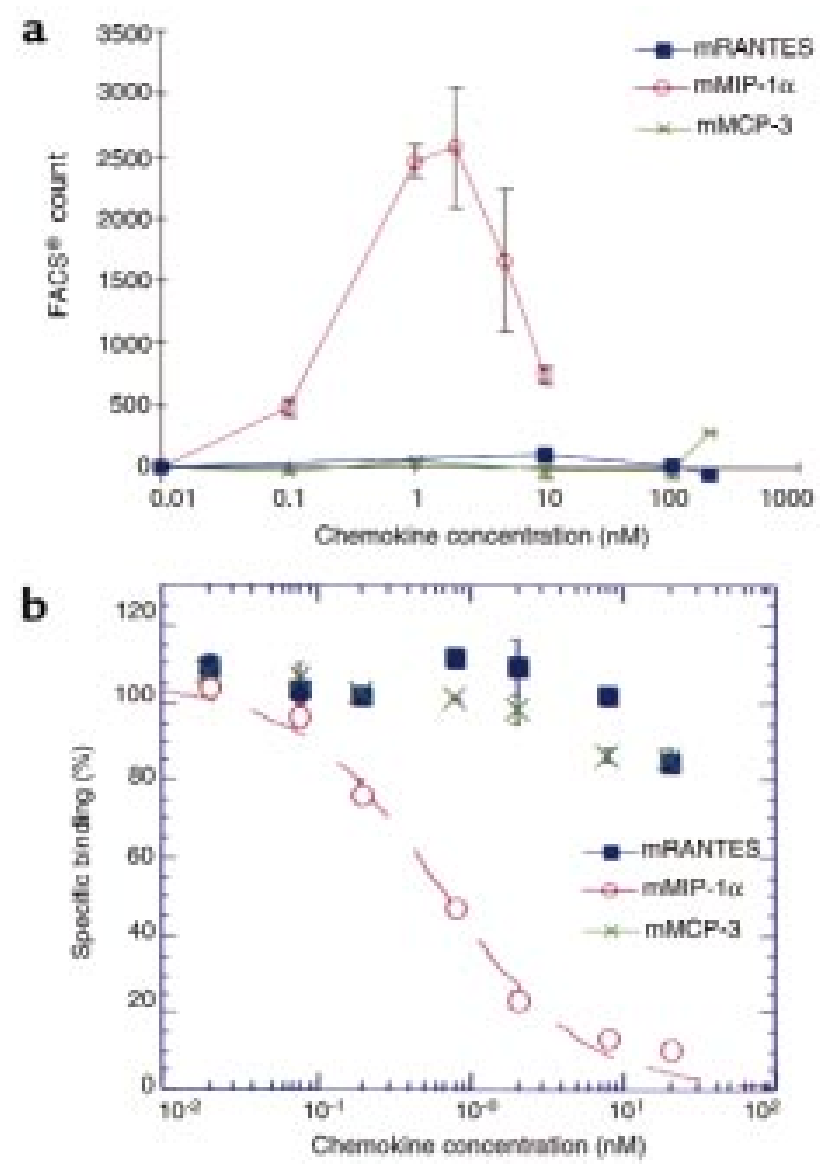

Figure 7

MIP- $1 \alpha$ is the major functional ligand of CCR 1 in the mouse, as shown by comparison with murine RANTES and MCP-3. (a) Representative experiment (performed 4 times) showing the chemotactic response of murine CCR 1 -transfected L1/2 cells to mouse MIP- $1 \alpha$ (filled squares) but not mouse RANTES or MCP-3. The latter chemokines were active as shown in control studies with mouse CCR5 and CCR3 transfectants, respectively (not shown). Transfectants were exposed to increasing doses of chemokine, and the chemotactic response was assessed by flow cytometric counting of $\mathrm{CCR} 1^{+}$cells in the lower well of the chemotaxis chamber. (b) Competitive binding assays showing that mouse MIP- $1 \alpha$, but not mouse RANTES or MCP-3, was able to competitively inhibit binding of radiolabeled human MIP- $\alpha$ to murine CCR 1 -transfected $\mathrm{L} 1 / 2$ cells (experiment performed 3 times). Results are presented as mean \pm SD.

of disease. Somewhat unexpectedly, when compared with $C C R 1^{+/+}$mice, $C C R 1^{-/-}$mice developed more severe renal disease in terms of impairment of renal function, protein excretion, and histologic injury, including glomerular crescent formation. Recent studies have convincingly demonstrated that glomerular crescent formation is the result of a Th1 nephritogenic immune response (26-28). For this reason, we compared the nature of the cell-mediated immune response in CCR1 $1^{-/-}$and $C C R 1^{+/+}$mice. By several criteria it appears that $C C R 1^{-/-}$mice have a more powerful Th1-deviated immune response than that found in $C C R 1^{+/+}$mice, including more robust DTH responses to sheep IgG, antigen-specific IgG2a isotype production, and en- hanced IFN- $\gamma$ production by splenic T cells. Data from 2 nonrenal models also indicate that CCR1 has a role in directing the cell-mediated immune response. In a Schistosoma mansoni-induced lung granuloma model in $\mathrm{CCR}^{-/-}$mice (23), Gao et al. noted enhanced antigenspecific production of IFN- $\gamma$ and reduced production of IL-4 by regional lymphoid cells, which is consistent with a Th1-predominant response. In addition, Kim et al. examined the effect of various chemokines on the immune response to DNA immunogens in mice (29). Coimmunization of the DNA vaccine construct with a cDNA expression cassette encoding MIP- $1 \alpha$ resulted in a Th2-polarized immune response (as determined by antigen-specific IgG isotype production), leading to the hypothesis that if this effect of MIP- $1 \alpha$ is mediated through CCR1, as it appears from our own data, then the absence of CCR1 might favor a Th1 phenotype.

Compared with control mice, CCR1 $1^{-/}$animals showed enhanced TNF- $\alpha$ and lymphotoxin mRNA expression in nephritic kidneys both early and late in the course of NTN, and in vitro CCR1 $1^{-/-}$splenocytes showed increased TNF- $\alpha$ production upon stimulation with LPS. Because TNF- $\alpha$ is a Th1-associated cytokine, the in vivo data may ultimately reflect the Th1-deviated response in $\mathrm{CCR}^{-/-}$mice. At the same time, the in vitro data leave open the possibility that CCR1 is involved in regulation of TNF- $\alpha$ production, potentially through an autocrine loop. In preliminary studies (data not shown), addition of CCR1 ligands did not diminish TNF- $\alpha$ production by LPS-stimulated mouse $C C R 1^{+/+}$macrophages, but further studies are necessary. Regardless of the mechanism responsible, TNF- $\alpha$ has been shown to play a critical role in the pathogenesis of glomerulonephritis (30), suggesting that its enhanced production in $\mathrm{CCR} 1^{-/}$mice could well contribute to the adverse outcome we observed.

The chemotaxis and binding studies show for the first time that in the mouse, RANTES and MCP-3 are not functional CCR1 ligands. This data helps to explain why the loss of CCR1 failed to prevent cell recruitment into the nephritic kidney, given the robust expression of RANTES and MCP-3 in this model. As in a previous report, MIP- $1 \alpha$ - the only functional ligand for murine CCR1 according to our studies - was expressed to only a minor extent in this model (11). This suggests that CCR1 is carried into the inflamed kidney by cells that are recruited through alternative chemotactic pathways (e.g., CCR2 or CCR5), highlighting the complexity and redundancy of this system. Taken with the functional effects of CCR1 deficiency in this system, the increased IFN- $\gamma$ and associated IgG2a humoral responses, and the enhanced production of TNF and related cytokines in $C C R 1^{-/-}$mice, our data suggest that the critical role of CCR1 in this model is not to promote chemotaxis but rather to facilitate and orchestrate the nature of cell-mediated immune responses. Caution is warranted in extrapolation of these data because findings from knockout mice do not necessarily predict events in wild-type animals in which the 
same pathway is blocked. However, in the absence of neutralizing antibodies or small molecules to inhibit CCR1 binding by its ligands in rodents, the data are both surprising and provocative.

In summary, this study shows the expression pattern of a number of chemokine receptors in a mouse model of crescentic glomerulonephritis, which will serve as a basis for future interventional studies. In addition, by examining the role of 1 of these prominently expressed chemokine receptors, CCR1, we have documented a redundant role in cell recruitment, but an unexpected and pathogenetically important role in modulating the effector phase of the immune response.

\section{Acknowledgments}

This work was supported in part by a grant from the National Institutes of Health to D.J. Salant (DK-30932).

1. Schreiner, G.F. 1991. The role of the macrophage in glomerular injury. Semin. Nephrol. 11:268-275.

2. Tipping, P.G., Huang, X.R., Qi, M., Van, G.Y., and Tang, W.W. 1998. Crescentic glomerulonephritis in CD4- and CD8-deficient mice. Requirement for CD4 but not CD8 cells. Am. J. Pathol. 152:1541-1548.

3. Rovin, B.H., and Phan, L.T. 1998. Chemotactic factors and renal inflammation. Am. J. Kidney Dis. 31:1065-1084.

4. Rovin, B.H., Rumancik, M., Tan, L., and Dickerson, J. 1994. Glomerular expression of monocyte chemoattractant protein- 1 in experimental and human glomerulonephritis. Lab. Invest. 71:536-542.

5. Tam, F.W., et al. 1996. Differential expression of macrophage inflammatory protein- 2 and monocyte chemoattractant protein- 1 in experimental glomerulonephritis. Kidney Int. 49:715-721.

6. Wu, X., et al. 1994. Cytokine-induced neutrophil chemoattractant mediates neutrophil influx in immune complex glomerulonephritis in rat. $J$. Clin. Invest. 94:337-344.

7. Natori, Y., Sekiguchi, M., Ou, Z., and Natori, Y. 1997. Gene expression of $\mathrm{CC}$ chemokines in experimental crescentic glomerulonephritis (CGN). Clin. Exp. Immunol. 109:143-148.

8. Tang, W.W., Yin, S., Wittwer, A.J., and Qi, M. 1995. Chemokine gene expression in anti-glomerular basement membrane antibody glomerulonephritis. Am. J. Physiol. 269:F323-F330.

9. Feng, L., Xia, Y., Yoshimura, T., and Wilson, C.B. 1995. Modulation of neutrophil influx in glomerulonephritis in the rat with anti-macrophage inflammatory protein-2 (MIP-2) antibody. J. Clin. Invest. 95:1009-1017.

10. Wu, X., Dolecki, G.J., Sherry, B., Zagorski, J., and Lefkowith, J.B. 1997. Chemokines are expressed in a myeloid cell-dependent fashion and mediate distinct functions in immune complex glomerulonephritis in rat. J. Immunol. 158:3917-3924.

11. Lloyd, C.M., et al. 1997. RANTES and monocyte chemoattractant protein-1 (MCP-1) play an important role in the inflammatory phase of crescentic nephritis, but only MCP-1 is involved in crescent formation and interstitial fibrosis. J. Exp. Med. 185:1371-1380.

12. Tesch, G.H., et al. 1999. Monocyte chemoattractant protein-1 promotes macrophage-mediated tubular injury, but not glomerular injury, in nephrotoxic serum nephritis. J. Clin. Invest. 103:73-80.

13. Murphy, P.M. 1994. The molecular biology of leukocyte chemoattractant receptors. Annu. Rev. Immunol. 12:593-633.

14. Bonecchi, R., et al. 1998. Differential expression of chemokine receptors and chemotactic responsiveness of type $1 \mathrm{~T}$ helper cells (Th1s) and Th2s. J. Exp. Med. 187:129-134.

15. Salant, D.J., and Cybulsky, A.V. 1988. Experimental glomerulonephritis. Methods Enzymol. 162:421-461.

16. Gerard, C., et al. 1997. Targeted disruption of the beta-chemokine receptor CCR1 protects against pancreatitis-associated lung injury. J. Clin. Invest. 100:2022-2027.

17. Hancock, W.W., Buelow, R., Sayegh, M.H., and Turka, L.A. 1998. Antibody-induced transplant arteriosclerosis is prevented by graft expression of anti-oxidant and anti-apoptotic genes. Nat. Med. 4:1392-1396.

18. Chomczynski, P., and Sacchi, N. 1987. Single-step method of RNA isolation by acid guanidinium thiocyanate-phenol-chloroform extraction. Anal. Biochem. 162:156-159.

19. Ponath, P.D., et al. 1996. Molecular cloning and characterization of a human eotaxin receptor expressed selectively on eosinophils. J. Exp. Med. 183:2347-2448

20. Clark-Lewis, I., et al. 1991. Chemical synthesis, purification, and characterization of two inflammatory proteins, neutrophil activating peptide 1 (interleukin-8) and neutrophil activating peptide. Biochemistry. 30:3128-3135.

21. Hebert, M.J., et al. 1998. Acute nephrotoxic serum nephritis in complement knockout mice: relative roles of the classical and alternate pathways in neutrophil recruitment and proteinuria. Nephrol. Dial. Transplant. 13:2799-2803.

22. Saleem, S., et al. 1998. IL-4 is an endogenous inhibitor of neutrophil influx and subsequent pathology in acute antibody-mediated inflammation. J. Immunol. 160:979-984.

23. Gao, J.L., et al. 1997. Impaired host defense, hematopoiesis, granulomatous inflammation and type 1-type 2 cytokine balance in mice lacking CC chemokine receptor 1. J. Exp. Med. 185:1959-1968.

24. Bozic, C.R., et al. 1994. The murine interleukin 8 type B receptor homologue and its ligands. Expression and biological characterization. J. Biol. Chem. 269:29355-29358.

25. Oda, T., et al. 1998. Cell-to-cell interaction is required to induce proteinuria in in-situ immune complex glomerulonephritis. J. Lab. Clin. Med. 132:112-123.

26. Kitching, A.R., Tipping, P.G., Mutch, D.A., Huang, X.R., and Holdsworth, S.R. 1998. Interleukin-4 deficiency enhances Th1 responses and crescentic glomerulonephritis in mice. Kidney Int. 53:112-118.

27. Kitching, A.R., Holdsworth, S.R., and Tipping, P.G. 1999. IFN-gamma mediates crescent formation and cell-mediated immune injury in murine glomerulonephritis. J. Am. Soc. Nephrol. 10:752-759.

28. Huang, X.R., Tipping, P.G., Shuo, L., and Holdsworth, S.R. 1997. Th1 responsiveness to nephritogenic antigens determines susceptibility to crescentic glomerulonephritis in mice. Kidney Int. 51:94-103.

29. Kim, J.J., et al. 1998. CD8 positive T cells influence antigen-specific immune responses through the expression of chemokines. J. Clin. Invest. 102:1112-1124.

30. Le Hir, M., Haas, C., Marino, M., and Ryffel, B. 1998. Prevention of crescentic glomerulonephritis induced by anti-glomerular membrane antibody in tumor necrosis factor-deficient mice. Lab. Invest. 78:1625-1631. 